\title{
Causes de contamination microbienne d'importance moyenne du lait dans un groupe de fermes de la région de Rennes
}

\author{
par \\ Christine PITON et J. RICHARD*
}

\section{INTRODUCTION}

Pour déterminer l'importance relative des différentes causes de contamination microbienne du lait à la ferme, une méthodologie particulière a été appliquée entre 1973 et 1976 dans une cinquantaine d'exploitations de la région de Rennes. Les quelques cas de contaminations importantes qui y ont été observés ont déjà fait l'objet d'un article dans ce journal [1]. Dans le cas le plus général, la contamination du lait était d'importance moyenne (de l'ordre de 50000 microorganismes totaux $/ \mathrm{ml}$ ) et résultait sans doute de plusieurs causes (mamelles insuffisamment lavées, matériel de traite en mauvais état ou mal nettoyé, refroidissement défectueux du lait); la contribution de chacune de ces causes était difficile à estimer car les déterminations directes de l'apport microbien par la peau des mamelles (traite avec du matériel stérile) et par le matériel de traite (traite avec mamelles soigneusement nettoyées) étant très lourdes à mettre en œuvre, ne pouvaient être appliquées que dans un petit nombre de fermes.

Le rinçage des installations de traite avec de l'eau stérile, dans des conditions rappelant celles de la traite, devrait permettre d'évaluer de façon relativement plus simple le pouvoir contaminant de ce matériel, étant donné que l'eau extrait autant de micro-organismes que le lait au cours de la traite [2]. Se basant sur la corrélation significative entre les résultats de l'examen bactériologique de l'eau de rinçage et du lait de tank, RICHARD [2] a pu faire une prédiction du niveau de contamination du lait par les machines à traire. Par différence, on devrait obtenir une estimation de l'apport par la peau des

* Laboratoire de Microbiologie Laitière et de Génie Alimentaire, I.N.R.A. C.N.R.Z. - 78350 Jouy-en-Josas. 
mamelles, dans le cas où la multiplication bactérienne dans le tank peut être écartée. Cependant, une étude récente de la cinétique d'extraction des micro-organismes par rinçage [3] permet théoriquement une nouvelle évaluation, plus précise de l'apport du matériel de traite et montre que cet apport est moins important que l'estimation proposée par RICHARD [2].

Il nous a donc paru maintenant possible de tenter, dans 14 fermes de la région de Rennes, une détermination de la part des deux causes de pollution bactérienne du lait les plus fréquentes : la peau des mamelles [4] et le matériel de traite.

\section{METHODES}

\section{Prédiction de l'apport du matériel de traite}

Les 14 installations de traite étudiées étaient nettoyées selon trois méthodes différentes : nettoyage classique deux fois par jour avec une solution chaude $\left(50^{\circ} \mathrm{C}-60^{\circ} \mathrm{C}\right)$ d'un détergent alcalin chloré, nettoyage à froid à l'aide d'une solution d'iodophore préparée une fois par jour [5], nettoyage à l'eau chaude acidifiée [6]. Les rinçages de ce matériel étaient réalisés selon la technique décrite par CHATELIN et RICHARD [1], une à deux fois par trimestre pendant 2 à 3 ans.

Nous avons mis en évidence par ailleurs [3] que les nombres de micro-organismes récoltés au cours de six à neuf rinçages successifs décroissent à peu près de la même manière quelle que soit l'installation de traite ; en outre leur évolution satisfait, en moyenne, un modèle mathématique simple qui permet, connaissant le niveau de population microbienne au second rinçage, de calculer le nombre de microorganismes récoltés par rinçage pendant un temps donné. Dans la mesure où l'eau extrait autant de micro-organismes que le lait [2], on peut en déduire le niveau de contamination du lait par le matériel. Pour ne pas risquer de faire une sous-évaluation de cette contribution, nous avons choisi un modèle qui nous donne la borne supérieure de cet apport.

Pour vérification, nous avons également réalisé dans quelques fermes (voir tab. 2), une détermination directe des apports microbiens par le matériel de traite et par la peau des mamelles.

\section{Détermination directe de l'apport du matériel de traite}

Les échantillons de lait étaient prélevés à la sortie de l'installation de traite, les mamelles étant, à cette occasion, soigneusement lavées (voir § 3) de façon à réduire au minimum la contamination par la peau des trayons. 


\section{Détermination directe de l'apport de la peau des mamelles}

Pour évaluer la contribution de la peau des trayons à la contamination du lait, le lait était récolté avec du matériel stérilisé à la vapeur. Une moitié du troupeau subissait un lavage des mamelles pratiqué habituellement par le producteur : une ou deux lavettes pour tout le troupeau, eau pas assez souvent renouvelée, lavage rapide ; pour l'autre moitié, les mamelles étaient très soigneusement nettoyées avec une solution détergente et des lavettes propres, puis rincées avec une solution d'eau de javel et essuyées.

\section{Numérations microbiennes}

Les échantillons d'eau de rinçage et de lait prélevé à la sortie de l'installation ou dans le tank (mélange de deux ou quatre traites) étaient conservés dans un mélange d'eau et de glace jusqu'à l'arrivée au laboratoire puis soumis aux dénombrements suivants :

- flore aérobie mésophile sur milieu Plate Count Agar (Difco 479) avec une incubation de $3 \mathrm{j}$ à $30^{\circ} \mathrm{C}$;

- flore psychrotrophe sur le même milieu avec une incubation de $10 \mathrm{j}$ à $7 \circ \mathrm{C}$;

- flore thermorésistante : après chauffage pendant $30 \mathrm{~min}$ à $63,5^{\circ} \mathrm{C}$ du lait ou du mélange volume à volume d'eau de rinçage et de lait écrémé stérile (Skim Milk Difco à 20 p. 100), dénombrement selon la méthode appliquée pour la flore totale.

\section{RESULTATS ET DISCUSSION}

Les résultats de l'examen bactériologique du lait de tank dans les fermes étudiées sont présentés dans le tableau 1, pour les trois groupes microbiens dénombrés et les trois méthodes de nettoyage des installations de traite. On n'observe pas, sur le plan de la qualité bactériologique du lait, de différences importantes entre ces trois méthodes. D'autre part, la pollution du lait ne peut être attribuée à une croissance bactérienne durant sa conservation puisque les nombres de micro-organismes totaux ou psychrotrophes du lait de deux traites ne diffèrent pas significativement de ceux du lait de quatre traites. Pour les micro-organismes thermorésistants, nous ne possédons que les résultats se rapportant à quatre traites mais il a été largement montré qu'ils ne se développent pas à basse température [7]. Cependant, on ne peut pas écarter une multiplication bactérienne pendant les premières heures de stockage, en raison d'un refroidissement trop lent ou différé [8]. 


\section{TABLEAU 1}

Examen bactériologique du lait prélevé dans les tanks de ferme pendant 3 ans (moyenne logarithmique du nombre de micro-organismes/ml)

\begin{tabular}{|c|c|c|c|c|c|}
\hline \multirow{2}{*}{ Méthode de nettoyage } & \multicolumn{2}{|c|}{ Flore totale } & \multicolumn{2}{|c|}{ Flore psychrotrophe } & \multirow{2}{*}{$\begin{array}{c}\text { Flore } \\
\text { thermorésistante } \\
\text { quatre traites }\end{array}$} \\
\hline & deux traites & quatre traites & deux traites & quatre traites & \\
\hline Classique (6 fermes) & 54000 (229) & $56000(69)$ & 7100 (176) & $7600(52)$ & $980(116)$ \\
\hline A froid (6 fermes) & $46000(342)$ & $47000(71)$ & $11000(270)$ & $10000(48)$ & $1900(206)$ \\
\hline Eau chaude acidifiée & & & & & \\
\hline - Ferme F & 26000 (19) & - & $2200(19)$ & - & 960 (19) \\
\hline - Ferme LEF 9 & - & 72000 (21) & - & 8800 (21) & $2100(21)$ \\
\hline
\end{tabular}




\section{TABLEAU 2}

Evaluation de l'apport en micro-organismes des différentes sources de contamination du lait

\begin{tabular}{|c|c|c|c|c|c|}
\hline \multirow{2}{*}{ Méthode de nettoyage } & \multirow{2}{*}{$\begin{array}{l}\text { Conditions } \\
\text { expérimentales }\end{array}$} & \multirow{2}{*}{$\mathrm{N}$} & \multicolumn{3}{|c|}{ Nombre de micro-organismes/ml (a) } \\
\hline & & & Flore totale & $\begin{array}{c}\text { Flore } \\
\text { psychrotrophe }\end{array}$ & $\begin{array}{c}\text { Flore } \\
\text { thermorésistante }\end{array}$ \\
\hline \multirow[t]{3}{*}{ Classique (6 fermes) } & Traite ordinaire (b) & 229 & 54000 & 7100 & 980 \\
\hline & Prédiction & 23 & 4700 & 480 & 820 \\
\hline & Traite soignée (c) & 4 & 9400 & 1100 & 460 \\
\hline \multirow[t]{3}{*}{ A froid (6 fermes) } & Traite ordinaire (b) & 342 & 46000 & 11000 & 1900 \\
\hline & Prédiction & 34 & 11000 & 3000 & 220 \\
\hline & Traite soignée (d) & 10 & 15000 & 3800 & 270 \\
\hline \multirow[t]{3}{*}{ Eau chaude acidifiée (ferme LEF 9) } & Traite ordinaire (b) & 21 & 72000 & 8300 & 2100 \\
\hline & Prédiction & 3 & 150 & 65 & 50 \\
\hline & Traite soignée & 1 & 9000 & 300 & 300 \\
\hline \multirow[t]{2}{*}{ Stérilisation à la vapeur (3 fermes) } & Traite ordinaire & 4 & 55000 & 2100 & 2500 \\
\hline & Traite soignée & 4 & 3500 & 280 & 26 \\
\hline \multicolumn{6}{|c|}{$\begin{array}{l}\text { (a) : moyenne géométrique des nombres de bactéries/r } \\
\text { (b) : lait pris dans le tank (mélange de deux traites). } \\
\text { (c et d) : lait récolté en bidons stériles dans respectivement }\end{array}$} \\
\hline
\end{tabular}


Le tableau 2 donne, pour les trois méthodes de nettoyage, la qualité bactériologique moyenne du lait (mélange de deux traites) avec lavage ordinaire des mamelles, celle-ci lorsque la contamination par la peau des trayons a été réduite au minimum (traite soignée) ainsi que la prédiction de l'apport maximum par le matériel de traite. Quels que soient la méthode de nettoyage et le groupe microbien, l'apport prédit du matériel de traite est très nettement inférieur aux résultats de dénombrements effectués sur le lait de tank. D'autre part, les résultats de numérations sur du lait produit lors de traites soignées sont également très nettement inférieurs à ces derniers. Cela prouve que la contamination par la peau des mamelles est largement dominante dans toutes ces fermes. L'importance de cette contamination est confirmée par les résultats de numérations, obtenus dans trois fermes, lors d'une traite ordinaire avec du matériel stérile (avant-dernière ligne du tableau 2). On observe que la contamination par la peau des mamelles affecte plus le niveau de la flore totale que celui des autres groupes microbiens, ce qu'avait déjà observé RICHARD [4] ; d'autre part, l'importance de cette contamination est très variable d'une ferme à l'autre ; ainsi les différences de qualité bactériologique du lait produit dans les fermes F et LEF 9 (tab. 1) ne sont explicables que par les apports par la peau des mamelles puisque dans ces fermes, la contribution du matériel de traite est très faible (moins de 1000 micro-organismes totaux $/ \mathrm{ml}$ pour les deux fermes). Le niveau de contamination du lait obtenu lors de traites soignées est, quelle que soit la méthode de nettoyage, supérieur à l'apport calculé de l'installation de traite. Cela est dû à l'ensemble des autres contaminations au cours de la traite (flore intramammaire, flore de la peau des mamelles qui n'a pu être éliminée malgré un lavage très soigné), dont on a pu estimer l'importance à l'aide d'une traite avec du matériel stérile (dernière ligne du tableau 2). Cependant dans la ferme LEF 9, si l'on retranche ce "bruit de fond " aux résultats correspondant à la traite soignée, on obtient des valeurs nettement supérieures à l'apport prédit du matériel ; cela est dû au fait que, dans cette ferme, le lavage des mamelles était difficile en raison d'une part de leur malpropreté et d'autre part, de la présence de nombreuses crevasses sur les trayons.

Par ailleurs, le niveau de la flore psychrotrophe dans le lait de tank est systématiquement supérieur à l'apport maximum observé pour les mamelles [4] augmenté de l'apport du matériel de traite ; cela peut s'expliquer par une contamination par le tank lui-même ou par une multiplication de cette flore au cours des premières heures de stockage.

\section{CONCLUSION}

Contrairement à ce qui est généralement admis, c'est la contamination par la peau des mamelles qui, dans l'ensemble de ces fermes, était la plus importante, et non celle par le matériel de traite. 
Cette contamination est cependant relativement peu importante mais elle peut avoir des conséquences technologiques considérables puisqu'il est bien connu que la peau des mamelles est la source principale de spores de Bacillus et de Clostridium [9, 10, 11]. D'autre part, l'apport de la peau des mamelles en micro-organismes psychrotrophes n'est pas négligeable, mais cette flore contient en fait peu de Pseudomonas, les seules bactéries psychrotrophes capables de se développer rapidement à basse température [12].

Cette étude a permis, par ailleurs, de réaliser une comparaison entre les différentes méthodes de nettoyage du matériel de traite sur la base de la contamination réelle du lait et non du " pouvoir maximum de contamination " pour ce matériel. C'est de loin la méthode à l'eau chaude acidifiée qui donne les meilleurs résultats ; cependant il paraît inutile de recommander cette méthode très coûteuse en énergie puisqu'elle n'a pas de répercussions sensibles sur la qualité bactériologique du lait. Par contre, bien que l'apport du matériel de traite soit plus important dans le cas du nettoyage à froid, cette méthode permettrait d'obtenir, dans les conditions de la pratique, du lait de très bonne qualité bactériologique (moins de 20000 microorganismes totaux $/ \mathrm{ml}$ si la contamination par les mamelles était réduite). Dans les quelques fermes où elle est appliquée sans discontinuité depuis 8 ans, le lait produit contient généralement moins de 50000 micro-organismes/ml. L'apport en micro-organismes psychrotrophes est nettement supérieur avec cette méthode de nettoyage qu'avec les autres mais on n'a pas observé de différences au niveau de la "qualité de conservation " du lait à basse température (niveau de population microbienne du lait après $4 \mathrm{j}$ de conservation à $4^{\circ} \mathrm{C}$ $5^{\circ}$ C) $[13]$.

\section{Résu mé}

L'importance de la contamination microbienne du lait par les mamelles et par le matériel de traite a été évaluée, sur une période de 3 ans, dans 14 fermes produisant du lait de qualité bactériologique moyenne (de 20000 à 100000 germes totaux/ml). Grâceà une évaluation précise de l'apport de la machine à traire, on a pu montrer que dans ces fermes, la peau des mamelles était de loin la source principale de contamination du lait. On a pu mettre aussi en évidence soit un apport non négligeable des tanks en bactéries psychrotrophes, soit une multiplication de cette flore dans les premières heures de conservation du lait.

\section{Su m mary}

\section{MAIN SOURCES OF BACTERIA IN MODERATELY CONTAMINED MILK ON FARMS IN RENNES AERA}

The part played by teat skin and milking machines in milk contamination was studied over a 3 year period on 14 farms producing milk in the range of $2.10^{4}-10^{5}$ bacteria/ml at the time of delivery. The milking 
machines contribution (MMC) was evaluated by rinsing them [2] and applying to the result of the second rinsing a «dilution factor» which takes in account the kinetic of the numbers of bacteria retrieved during consecutive rinsing and during milking $[2,3]$. The mean MMC for total counts was $4,700 / \mathrm{ml}$ for hot cleaning, $11,000 / \mathrm{ml}$ for cold cleaning and $<1,000 / \mathrm{ml}$ for BAW [5]. The teat skin was found as the major source of bacteria in all farms. The psychrotrophic count in milk was not accounted for by contamination by the milking machine and the teat skin. A contamination by the tank or a multiplication of this flora during the first hours of storage might occur.

Reçu pour publication en novembre 1981.

\section{Bibliographie}

[1] Chatelin (Y.M.) et Richard (J.) (1981). - Etude de quelques cas de contaminations microbiennes importantes du lait à la ferme. Le Lait, 61, 80-94.

[2] RichaRD (J.) (1981). - Bacteriological examination of pipeline milking machines by rinsing the entire system. Journal of Applied Bacteriology, $50,433-442$.

[3] Piton (Christine) and Richand (J.). (1982). - A kinetic study of the retrieval of bacteria from milking machine by rinsing the entire system with sterile water. Journal of Applied bacteriology (submitted for publication).

[4] RICHARD (J.) (1978). - Contamination microbienne du lait de vache par la peau des mamelles. XX ${ }^{\mathrm{e}}$ Congrès International de Laiterie, Paris, 92-93.

[5] Richard (J.) (1981). - Microbiological aspects of cold cleaning with an iodophor of milk pipeline installations. Journal of Applied Bacteriology, 50, 229-238.

[6] Clough (P.), AKam (D.) and Cant (D.) (1965). - Circulation cleaning of pipeline milking machines with boiling water. NIRD paper $\mathrm{n}^{\circ} 2939$.

[7] Thomas (S. B.), Egdell (J. W.), ClegG (L. F. L.) and Cuthbert (W. A.) (1950). Thermoduric organisms in milk. Part I. A review of the litterature. Proceeding of the Society for Applied Bacteriology, 13, 27-64.

[8] Mourgues (R.), Vassal (L.) et Auclair (J.) (1967), - Influence de la vitesse de refroidissement et de la température de conservation en tanks de ferme sur le développement de la flore microbienne du lait cru. Congrès International du froid, Madrid, 4-36, 1-8.

[9] Underwood (H. M.), Mc Kinnon (C. H.), Davies (F. L.) and Cousins (C. M.) (1974). - Sources of Bacillus spores in raw milk. XIX ${ }^{\bullet}$ Congrès International de Laiterie, B6, 406-407.

[10] Labussière (J.), Richard (J.), Combaud (J. F.) et de la Chevalerie (F. A.) (1976). - Suppression du massage et du lavage de la mamelle chez les vaches laitières. Effets sur les caractéristiques de traite et sur la qualité bactériologique du lait. Annales de Zootechnie, 25, 551-565.

[11] Bergère (J. L.), Gouet (P.), Hermier (J.) et Mocouot (G.) (1968). — Les Clostridium du groupe butyrique dans les produits laitiers. Annales de l'Institut Pasteur de Lille, XIX, 41-54.

[12] RICHARD (J.) (1982). - Taxonomy and ecology of raw milk psychrotrophic Pseudomonas. Psychrotrophic microorganisms in spoilage and pathogenicity, XI ICFMH Symposium Aalborg, 1980, in Academic Press INC London (sous presse).

[13] RichaRd (J.) (1981). - Influence de diverses méthodes de nettoyage des machines à traire sur la « qualité de conservation » du lait cru à basse température. Le Lait, 61, 354-369. 